\title{
A prospective comparative study of surgical release of carpal tunnel syndrome with and without flexor tenosynovectomy
}

\author{
Niraj Ranjeet, Pabin Thapa, Krishna Sapkota, Pratyenta Raj Onta, Upendra Jung Thapa, \\ Krishna Wahegoankar
}

Associate Professor, Department of Orthopedics, Manipal Teaching Hospital, Pokhara, Nepal

Background: Carpal tunnel syndrome (CTS) is a common condition causing hand pain and numbness. The rationale of tenosynovectomy is to remove the flexor tenosynovium that is contributing to increased volume within the carpal canal, thus removal of this tenosynovium should give more space for the median nerve and there by relief from symptoms. Aims and Objectives: The main objective of our study was to assess the role of routine flexor tenosynovectomy in the treatment of CTS. Materials and Methods: Eighty-six hands with CTS were randomized into two groups, either to carpal tunnel release with or without flexor tenosynovectomy. All patients were requested to fill up the self-administered questionnaire for the assessment of symptom severity and functional status, examined for 2-point discrimination, grip and pinch strength, scar tenderness and patient satisfaction score preoperatively, 2 weeks, 1 month, and 6 months follow-up. Results: After the surgical release, both groups improved significantly with respect to symptom severity and functional status, 2-point discrimination, grip and pinch strength, scar tenderness and patient satisfaction score but there was no significant difference between the two groups. There were two cases of post-operative wound infection and one case of mild reflex sympathetic dystrophy. Conclusion: We conclude that routine flexor tenosynovectomy during surgical release of carpal tunnel in patients with CTS does not provide any added benefit nor does it increase any morbidity.

Access this article online

Website:

http://nepjol.info/index.php/AJMS DOI: 10.3126/ajms.v13i1.39464

E-ISSN: 2091-0576

P-ISSN: 2467-9100

Copyright (c) 2022 Asian Journal of Medical Sciences

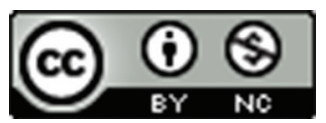

This work is licensed under a Creative Commons Attribution-NonCommercial 4.0 International License.

Key words: Carpal tunnel syndrome; Flexor tendon; Tenosynovectomy

\section{INTRODUCTION}

Carpal tunnel syndrome (CTS) still remains a disabling and puzzling condition commonly presented to orthopedic surgeons. The American Academy of Orthopedic Surgeons Clinical Guidelines defines CTS as a symptomatic mononeuropathy or radiculopathy because of compression of the median nerve at the level of the wrist. ${ }^{1,2}$ CTS is the most common form of median nerve entrapment, ${ }^{3,4}$ accounting for about $90 \%$ of all entrapment neuropathies ${ }^{5}$ and about $1-3.8 \%$ of the general population. ${ }^{6,7}$ An entrapment neuropathy is a chronic focal compressive neuropathy caused by an increase in pressure inside the fibro-osseous anatomical structures. ${ }^{8}$ Physiological evidence indicates increased pressure within the carpal tunnel, and therefore decreased function of the median nerve at that level. ${ }^{9}$

According to the biomechanical and histological findings, the most characteristic histological finding is noninflammatory fibrosis and thickening of the subsynovial connective tissue. It is assumed that trauma or disease of the synovium of the flexor tendons within the carpal tunnel because of aging process or forceful or repetitive movement may lead to degeneration of the median nerve. This ultimately increases the volume of the carpal tunnel contents and eventually compresses the median nerve within the tunnel and results in CTS. ${ }^{10}$ 
The management of CTS in recent practice depends on various factors, such as the severity of the symptoms, the stage of the disease, and the patient's preference. The initial stage of CTS is managed conservatively by analgesics, wrist splints, and oral or local injection of corticosteroids into the carpal tunnel. ${ }^{11}$ When conservative treatment fails, surgical treatment is considered. In the recent literature, surgical treatment has been reported to be more effective than other conservative treatment. ${ }^{10,11}$ Surgical decompression of the median nerve by division of the transverse carpal ligament (TCL), which is accepted as the most reliable procedure, is the treatment of choice and results in excellent results in almost $75 \%$ of the patients. ${ }^{12-14}$ In contrast to the theory that idiopathic CTS is caused by chronic tenosynovitis of the flexor tendons, signs of acute or chronic inflammation have rarely been found in histopathologic studies of the flexor tenosynovium of patients who were treated with surgical release of CTS. ${ }^{15-21}$ In addition, the benefit of flexor tenosynovectomy during Carpal Tunnel Release (CTR) for the treatment of idiopathic CTS is also undetermined.

There are many studies favoring and disfavoring tenosynovectomy at the time of CTR. Some studies believe a decrease in both carpal tunnel volume and adhesions contributes to the benefits and also indicate that because of significant decrease in pillar pain, a flexor tenosynovectomy in the treatment of CTS would likely benefit patients who use the palm of the hand in heavy manual or highly repetitive work by allowing them to return to regular duty sooner. ${ }^{22-24}$ However, other studies observed neither an added benefit nor an increased rate of morbidity in association with the flexor tenosynovectomy at the time of CTR. Furthermore, they could not identify any clinical correlations that might predict which patient would benefit from flexor tenosynovectomy on the basis of gross intraoperative evaluation of the flexor tenosynovium. ${ }^{18,25}$

Thus, the purpose of the present study was: (1) To assess the role of flexor tenosynovectomy in the operative treatment of CTS on the basis of clinical outcome measures, and (2) to assess whether the gross appearance of the flexor tenosynovium correlates with pre-operative or postoperative symptoms or function to determine whether a group of patients who might benefit from flexor tenosynovectomy could be identified intraoperatively.

\section{Aims and objectives}

The purpose of the present study was: (1) To assess the role of flexor tenosynovectomy in the operative treatment of CTS on the basis of clinical outcome measures, and (2) to assess whether the gross appearance of the flexor tenosynovium correlates with pre-operative or postoperative symptoms or function to determine whether a group of patients who might benefit from flexor tenosynovectomy could be identified intraoperatively.

\section{MATERIALS AND METHODS}

All patients with features of CTS coming to Manipal Teaching Hospital, Department of Orthopedics between July 1, 2019, and December 31, 2020, were enrolled in our study. There were 86 hands in 78 patients (18 males and 60 females) in our study with a mean age of 56.4 (46-70 years). The syndrome was bilateral in eight patients. The diagnosis of CTS was made on the clinical basis of paresthesia, pain, and/or weakness in the distribution of the median nerve at palm and hand. The most common presenting symptoms included pain $(n=58,67.44 \%)$, paresthesia $(n=65 ; 75.58 \%)$, and weakness $(\mathrm{n}=21 ; 24.41 \%)$. All patients were examined for manual muscle-testing, presence of visible thenar atrophy, evaluation of 2-point discrimination, and SemmesWeinstein monofilament testing. The provocative tests (Tinel and Phalen) were tested in all patients to assist in the diagnosis. ${ }^{26}$ The criteria for the diagnosis of CTS included a history of paresthesia in the median-nerve distribution, nocturnal hand pain, positive findings on physical examination, and a positive electrophysiologic study. The criteria for the electrophysiologic diagnosis included a median distal motor latency of $>4.5 \mathrm{~ms}$, a median sensory nerve conduction velocity of $<50 \mathrm{~m} / \mathrm{s}$ across the carpal tunnel, or a lumbrical/interosseous peak latency difference of $>0.5 \mathrm{~ms}$. Electrophysiologic studies of the thenar muscles were utilized to demonstrate signs of denervation and to measure the severity of median-nerve compression. All patients included in the study met the criteria for CTS by American Association of Electrodiagnostic Medicine Diagnostic. ${ }^{27-29}$ Standard radiographs of the hand and wrist were taken for all the patients to rule out any osseous abnormalities. Our exclusion criteria included patients who did not consent to the study, patients with evidence of infection, recurrent CTS, inflammatory arthropathy, peripheral neuropathy, pregnancy, thyroid abnormalities, acromegaly, or any tumors. Patients $<18$ years of age or more than 75 years were also excluded, to meet the guidelines of the human subject committees. Informed consent for the study was obtained from each patient. Surgery was indicated to all patients who had failure or who refused conservative management or features of thenar atrophy or denervation. Patients were randomized using a table of random numbers to either CTR with or without flexor tenosynovectomy using opaque sealed envelope system with 43 patients in each group.

\section{CTR}

All the surgeries were performed by a single surgeon in the operating room on an outpatient basis using local anesthesia ( $2 \%$ lidocaine). In both techniques, adequate anesthesia was confirmed before surgery. A curvilinear 
incision 2-3 $\mathrm{mm}$ ulnar to and parallel with the thenar crease is made beginning just proximal to Kaplan's line (a line drawn from the apex of the interdigital fold between the thumb and index finger, toward the ulnar side of the hand and parallel to the proximal palmar crease, and passing $4.0-5.0 \mathrm{~mm}$ distal to the pisiform bone) and stopping short of the volar flexion crease of the wrist. After blunt dissection, the TCL was released and the gross appearance of the Median Nerve and the flexor tenosynovium was observed. The proper release of carpal tunnel was confirmed by "Fat pad" and "Little finger pulp" signs. ${ }^{30}$ Flexor tendons were then retracted radially to inspect the floor of the canal for ganglia, calcifications or osteophytes. The tenosynovium was than subjectively graded as mild, moderate, or severe on the basis of the amount of tenosynovium present, its apparent thickness, and the degree of hyperemia. In the group with tenosynovectomy, the visible tenosynovium that could be withdrawn into the incision by pulling the flexor tendons into the wound was excised, with care taken to protect the median nerve. We observed that the tenosynovium was more abundant on the flexor digitorum superficialis tendons than on the flexor digitorum profundus tendons. The tenosynovium specimens were sent to the pathology department for histopathological examination. In both the groups, the wound was then washed, sutured with 3-0 nylon sutures and a bulky dressing was placed. Splints were not used for any patients.

\section{Post-operative regimen}

The patients were allowed to use the hand for light activities for the initial 2 weeks. The dressings and sutures were removed at 2 weeks and the patients were instructed for hand physiotherapy. Patients were then followed up at 1 month and 6 months after surgery. On each follow-up, patients were inquired and evaluated about postoperative pain, presence of infection, paresthesia, grip and pinch strength, 2-point discrimination, and any complications. Preoperatively and at each follow-up the patients were requested to complete a validated self-administered questionnaire for the scaled assessment of the severity of symptoms and functional status as described by Levine et al., ${ }^{31}$ The responses on this questionnaire are assigned a score of $1-5$ points, with 1 point as the best score and 5 points as the worst score.

\section{Statistical analysis}

For the study design to achieve at least $95 \%$ power with the use of an unpaired $t$ test with a large effect size of 0.8 and an alpha $(\alpha)$ of 0.05 , it was determined that at least 38 samples were required in each group. With a total of 43 samples in each group in our study, these numbers were sufficient for the analysis. Statistical analysis was performed with use of the SAS statistical package (SAS Institute, Cary, North Carolina) and included paired and unpaired t testing, multiple analysis of variance, and determination of Pearson correlation coefficients.

\section{RESULTS}

There were 43 patients in each group of CTR with and without tenosynovectomy. There were no significant differences between the two groups with respect to age, gender, handedness, duration of symptoms, electrodiagnostic findings, or bilaterality (Table 1).

On physical examination preoperatively, the Phalen sign was positive in 55 hands $(63.95 \%)$, the Tinel sign was positive in 62 hands $(72.1 \%)$, and thenar atrophy in 28 hands $(32.55 \%)$. The average 2 -point discrimination was $6 \mathrm{~mm}$ and on Semmes-Weinstein monofilament testing, $10(11.62 \%)$ patients showed diminished sensitivity to light touch, 31 (36.04\%) exhibited diminished protective sensation, and $26(30.23 \%)$ showed a loss of protective sensibility.

The grip strength of the involved and uninvolved hands was measured with use of all five settings of the Jamar dynamometer (Asimov Engineering, Los Angeles, California). The maximum grip was used for the statistical analysis. Preoperatively, the sensory latency averaged $4.2 \mathrm{msec}$ and the motor latency averaged $5.3 \mathrm{msec}$ in the group with synovectomy and the sensory latency averaged $4.4 \mathrm{msec}$ and the motor latency averaged $5.6 \mathrm{msec}$ in the group without synovectomy.

Compared to the pre-operative status, the symptomseverity and functional assessment scores improved both in the group treated with tenosynovectomy $(\mathrm{P}<0.0002$ and $\mathrm{P}=0.05$, respectively; paired t-test) and in the group treated without tenosynovectomy $(\mathrm{P}=0.0003$ and $\mathrm{P}=0.004$,

\begin{tabular}{lcc} 
Table 1: Demographic variables & \\
\hline Variables & $\begin{array}{c}\text { With } \\
\text { tenosynovectomy }\end{array}$ & $\begin{array}{c}\text { Without } \\
\text { tenosynovectomy }\end{array}$ \\
\hline Sex & & \\
$\quad$ Male & 10 & 12 \\
Female & 33 & 31 \\
Age in years & & \\
$\quad$ Mean (56.4) & $49-65$ & $46-70$ \\
Clinical & & \\
presentation & & \\
Pain & $28(65.11 \%)$ & $30(69.76 \%)$ \\
Paresthesia & $30(69.76 \%)$ & $35(81.39 \%)$ \\
Weakness & $7(16.27 \%)$ & $14(32.55 \%)$ \\
Phalen's test & $30(69.76 \%)$ & $25(58.13 \%)$ \\
Tinel's sign & $34(79.06 \%)$ & $28(65.11 \%)$ \\
Thenar atrophy & $12(27.90 \%)$ & $16(37.20 \%)$ \\
\hline
\end{tabular}


respectively; paired t-test). Specifically, the mean symptomseverity score improved from $3.2 \pm 0.78$ to $1.5 \pm 0.62$ points in the group treated with tenosynovectomy and from $2.8 \pm 0.58$ to $1.4 \pm 0.63$ points in the group treated without tenosynovectomy. The mean functional status score improved from $3.1 \pm 0.62$ to $1.5 \pm 0.68$ points in the group treated with tenosynovectomy and from $2.9 \pm 0.57$ to $1.7 \pm 0.48$ points in the group treated without tenosynovectomy. At 6 months follow-up, there were no significant differences between the two groups with regard to the symptom-severity or functional status scores $(\mathrm{P}=0.64$ and $\mathrm{P}=0.52$, respectively; unpaired $\mathrm{t}$-test). There were no differences between the two groups with regard to finger motion, wrist motion, grip strength, or scar sensitivity.

The tenosynovium in the operating room were graded as mild in 11 wrists, moderate in 53 wrists, and severe for 22 wrists. However, on histologic analysis, the tenosynovium was graded as mild in 14 wrists, moderate 58 wrists, and severe in 14 wrists. Statistical analysis showed no significant correlation between the gross intraoperative grading and the histologic grading $(r=0.34)$. However, majority of the specimen $(\mathrm{n}=81,94.18 \%)$ demonstrated an absence of acute inflammation as indicated by the absence of a neutrophilic exudate in the specimens.

\section{Patient's satisfaction score}

The overall patient satisfaction score was quite good. At 2 weeks follow-up, it was $3.9 \pm 0.13$ in the tenosynovectomy group and $4.1 \pm 0.14$ in the group without tenosynovectomy, which was not significant difference $(\mathrm{P}<0.35)$. At 6 months follow-up, the patient satisfaction scores were $4.6 \pm 0.24$ and $4.8 \pm 0.18$ in the group with and without tenosynovectomy, which was not significant.

\section{Evaluation of sensation}

Before surgery, the mean score for the Semmes-Weinstein monofilament evaluation of the median nerve distribution was 4.64 and 4.83 in the group with and without tenosynovectomy. At 2 weeks follow-up, the sensation improved significantly in both groups $(\mathrm{P}<0.05)$ but there was no significant difference between the two groups $(\mathrm{P}=0.32)$. At 6 months follow-up, the sensibility was 3.12 and 2.94 in the group with and without tenosynovectomy, which was not significant.

\section{Scar sensitivity}

Preoperatively, the group with and without tenosynovectomy could withstand loads of $3.12 \mathrm{~kg}$ and 3.27 without discomfort. At 2 weeks follow-up, they could tolerate only 1.96 and $2.05 \mathrm{~kg}$ respectively which was significant as compared to pre-operative status $(\mathrm{P}<0.05)$. At 6 months follow-up, they were able to tolerate 2.93 and $2.98 \mathrm{~kg}$, respectively, which was not significant.
Grip and pinch strength

Preoperatively, the group with and without tenosynovectomy had the grip strength of $31.2 \mathrm{~kg}$ and 32.4, respectively. At 2 weeks follow-up, the grip strength decreased significantly in both groups (17.4 kg and $18.3 \mathrm{~kg})(\mathrm{P}<0.05)$. At 6 months follow-up, the grip strength increased to $28.5 \mathrm{~kg}$ and $29.8 \mathrm{~kg}$ respectively, which was not significant.

Similarly, preoperatively, the group with and without tenosynovectomy had the pinch strength of $7.2 \mathrm{~kg}$ and $7.4 \mathrm{~kg}$, respectively. At 2 weeks follow-up, the pinch strength decreased significantly in both groups $(3.45 \mathrm{~kg}$ and $3.82 \mathrm{~kg}$ ) $(\mathrm{P}<0.05)$. At 6 months follow-up, the pinch strength increased to $6.85 \mathrm{~kg}$ and $6.92 \mathrm{~kg}$, respectively, which was not significant.

\section{Complications}

One of the patients in the tenosynovectomy group had symptoms consistent with reflex sympathetic dystrophy, redness, swelling, and increased sweating. The symptoms were mild and resolved after physiotherapy. There were two cases of wound infections post operatively ( 1 in each group) which resolved with local wound care and oral antibiotics. None of the patients in either group had any nerve or artery injury (Table 2).

\section{DISCUSSION}

The cause of CTS has been proposed as the result of either an increase in the volume of the contents within the canal or a decrease in the size of the carpal canal. The previous studies have shown no significant differences with regard to the thickness, histological characteristics, and biomechanical behavior of the TCL between patients with and without CTS. 22,32 Many authors believe that CTS is caused by an increase in the volume of contents within the carpal tunnel because of tenosynovium, lumbrical, or superficialis muscles or is the result of swelling within the tunnel that is caused by either internal or external pressure, that leads to chronic tenosynovial thickening and fibrosis. ${ }^{20}$

Many authors relate a nonspecific chronic tenosynovitis as a cause of this syndrome; however, the histologic studies have failed to demonstrate any inflammatory changes in the removed tenosynovium but rather non-specific fibrous changes and edema are noted. ${ }^{15,20-22}$ Our study also demonstrated no correlation between the gross and histologic appearance of the tenosynovium before and after surgery.

The main objective of our study was to assess the role of routine flexor tenosynovectomy in the treatment of CTS. The rationale of tenosynovectomy is to remove the flexor tenosynovium that is contributing in increasing the volume within the carpal canal, thus removal of this tenosynovium should give more space for the median nerve and there by relief from symptoms. Previously, tenosynovectomy 


\begin{tabular}{lccccc} 
Table 2: Outcome measures & \multicolumn{1}{l}{} \\
Outcome variables & Tenosynovectomy & Preoperative & $\mathbf{2}$ weeks follow-up & $\mathbf{1}$ month follow-up & $\mathbf{6}$ months follow-up \\
\hline Symptom-severity scores & + & $3.2 \pm 0.78$ & $1.5 \pm 0.62$ & $1.4 \pm 0.37$ & $1.1 \pm 0.21$ \\
& - & $2.8 \pm 0.58$ & $1.4 \pm 0.63$ & $1.3 \pm 0.25$ & $1.1 \pm 0.37$ \\
Functional assessment scores & + & $3.1 \pm 0.62$ & $1.5 \pm 0.68$ & $1.4 \pm 0.48$ & $1.1 \pm 0.33$ \\
Patient's satisfaction score & - & $2.9 \pm 0.57$ & $1.7 \pm 0.48$ & $1.5 \pm 0.52$ & $1.2 \pm 0.28$ \\
& + & & $3.9 \pm 0.13$ & $4.1 \pm 0.15$ & $4.6 \pm 0.24$ \\
Semmes-Weinstein & - & & $4.1 \pm 0.14$ & $4.2 \pm 0.11$ & $4.8 \pm 0.18$ \\
monofilament test (in mm) & + & 4.64 & 4.21 & 3.92 & 3.12 \\
Scar sensitivity (in kg) & - & 4.83 & 4.06 & 3.64 & 2.94 \\
Grip strength (in kg) & + & 3.12 & 1.96 & 2.15 & 2.93 \\
& - & 3.27 & 2.05 & 2.34 & 2.98 \\
Pinch strength (in kg) & + & 31.2 & 17.4 & 21.1 & 28.5 \\
Complications & - & 32.4 & 18.3 & 4.32 & 29.8 \\
& + & 7.2 & 3.45 & 4.51 & 6.85 \\
\end{tabular}

with release of TCL has been indicated for conditions like granulomatous infection, rheumatoid arthritis, gout, and amyloid deposition in patients receiving dialysis.

In the study by Freshwater, they compared two groups of patients with CTS, one group with a CTR alone, and the other group with CTR, tenosynovectomy, external neurolysis of the median nerve, and instillation of triamcinolone acetonide. The authors found no significant difference between the two groups with regard to signs, symptoms, electromyographic data or complications. ${ }^{33}$

In our study, we found no difference between the two groups with regards to preoperative demographic, symptom-severity and functional assessment scores, patient satisfaction score, Semmes-Weinstein monofilament evaluation, scar tenderness, grip, and pinch strength and complications. In our study, we choose a self-administered questionnaire which is a validated tool specifically designed to assess patients with CTS. ${ }^{31}$

\section{Limitations of the study}

One of the limitation in our study was a lack of standard evaluation form in our country. A larger population and a longer follow-up would have been better to evaluate the outcome.

\section{CONCLUSION}

Our results favor the theories by Phalen in his landmark article of 1966, which suggested that "thickening or fibrosis of the flexor synovialis within the carpal tunnel was the most common cause of the syndrome, and routine synovectomy is not advisable." 34 Thus, we conclude that routine flexor tenosynovectomy during surgical CTR in patients with CTS does not provide any added benefit nor does it increase any morbidity. Thus, tenosynovectomy is not recommended as it only increases the intraoperative time and blood loss.

\section{ACKNOWLEDGMENT}

None.

\section{REFERENCES}

1. Alfonso $\mathrm{C}$, Jann $\mathrm{S}$, Massa $\mathrm{R}$ and Torreggiani A. Diagnosis, treatment and follow-up of the carpal tunnel syndrome: A review. Neurol Sci. 2010;31(3):243-252.

https://doi.org/10.1007/s10072-009-0213-9

2. American Academy of Orthopaedic Surgeons Work Group Panel. Clinical Guidelines on Diagnosis of Carpal Tunnel Syndrome; 2007.

3. Padua L, LoMonaco M, Padua R, Gregori B and Tonali P. Neurophysiological classification of carpal tunnel syndrome: Assessment of 600 symptomatic hands. Ital J Neurol Sci. 1997;18(3):145-150. https://doi.org/10.1007/BF02048482

4. Lo SL, Raskin K, Lester $\mathrm{H}$ and Lester B. Carpal tunnel syndrome: A historical perspective. Hand Clin. 2002;18(2):211-217. https://doi.org/10.1016/s0749-0712(01)00014-2

5. Pfeffer GB, Gelberman RH, Boyes JH and Rydevik B. The history of carpal tunnel syndrome. J Hand Surg Br. 1988;13(1):28-34. https://doi.org/10.1016/0266-7681(88)90046-0

6. Atroshi I, Gummesson C, Johnsson R, Ornstein E, Ranstam J and Rosén I. Prevalence of carpal tunnel syndrome in a general population. JAMA. 1999;282(2):153-158. https://doi.org/10.1001/jama.282.2.153

7. Patiala H, Rokkanen $\mathrm{P}$, Kruuna $\mathrm{O}$, Taponen $\mathrm{E}$, Toivola $\mathrm{M}$ and Häkkinen V. Carpal tunnel syndrome. Anatomical and clinical investigation. Arch Orthop Trauma Surg. 1985;104(2):69-73. https://doi.org/10.1007/BF00454239

8. Aroori S and Spence RA. Carpal tunnel syndrome. Ulster Med J. 2008;77(1):6-17.

9. Dyck PJ, Thomas PK and Burns TM. Mechanisms of Acute and Chronic Compression Neuropathy in Peripheral Neuropathy. $4^{\text {th }}$ ed. Amsterdam: Elsevier; 2005. p. 1391-1402.

10. Verdugo RJ, Salinas RA, Castillo JL and Cea JG. Surgical versus non-surgical treatment for carpal tunnel syndrome. Cochrane Database Syst Rev. 2008;2008(4):CD001552. https://doi.org/10.1002/14651858.CD001552.pub2 
11. Piazzini DB, Aprile I, Ferrara PE, Bertolini C, Tonali P, Maggi L, et al. A systematic review of conservative treatment of carpal tunnel syndrome. Clin Rehabil. 2007;21(4):299-314.

https://doi.org/10.1177/0269215507077294

12. Bland JD. Treatment of carpal tunnel syndrome. Muscle Nerve. 2007;36(2):167-171.

https://doi.org/10.1002/mus.20802

13. Kim PK, Lee HJ, Kim TG and Jeon IH. Current approaches for carpal tunnel syndrome. Clin Orthop Surg. 2014;6(3):253-257. https://doi.org/10.4055/cios.2014.6.3.253

14. Hulsizer DL, Staebler MP, Weiss AP and Akelman E. The results of revision carpal tunnel release following previous open versus endoscopic surgery. J Hand Surg Am. 1998;23(5):865-869. https://doi.org/10.1016/S0363-5023(98)80164-0

15. Faithfull DK, Moir $\mathrm{DH}$ and Ireland J. The micropathology of the typical carpal tunnel syndrome. J Hand Surg Br. 1986;11(1):131-132. https://doi.org/10.1016/0266-7681(86)90035-5

16. Stevens JC, Beard CM, O'Fallon WM and Kurland LT. Conditions associated with carpal tunnel syndrome. Mayo Clin Proc. 1992;67(6):541-548.

https://doi.org/10.1016/s0025-6196(12)60461-3

17. Neal NC, McManners $\mathrm{J}$ and Stirling GA. Pathology of the flexor tendon sheath in the spontaneous carpal tunnel syndrome. J Hand Surg Br. 1987;12(2):229-232.

https://doi.org/10.1016/0266-7681(87)90020-9

18. Schuind $F$, Ventura $M$ and Pasteels JL. Idiopathic carpal tunnel syndrome: Histologic study of flexor tendon synovium. J Hand Surg Am. 1990;15(3):497-503. https://doi.org/10.1016/0363-5023(90)90070-8

19. Fuchs PC, Nathan PA and Myers LD. Synovial histology in carpal tunnel syndrome. J Hand Surg Am. 1991;16(4):753-758. https://doi.org/10.1016/0363-5023(91)90208-s

20. Lluch AL. Thickening of the synovium of the digital flexor tendons: Cause or consequence of the carpal tunnel syndrome? J Hand Surg Br. 1992;17(2):209-212. https://doi.org/10.1016/0266-7681(92)90091-f

21. Kerr CD, Sybert DR and Albarracin NS. An analysis of the flexor synovium in idiopathic carpal tunnel syndrome: Report of 625 cases. J Hand Surg Am. 1992;17(6):1028-1030. https://doi.org/10.1016/s0363-5023(09)91053-x

22. Nakamichi $\mathrm{K}$ and Tachibana S. Histology of the transverse carpal ligament and flexor tenosynovium in idiopathic carpal tunnel syndrome. J Hand Surg Am. 1998;23(6):1015-1024. https://doi.org/10.1016/s0363-5023(98)80009-9

23. Lynn DK. A comparison of flexor tenosynovectomy, open carpal tunnel release, and open carpal tunnel release with flexor tenosynovectomy in the treatment of carpal tunnel syndrome.
Plast Reconstr Surg. 2004;113(7):2020-2029. https://doi.org/10.1097/01.prs.0000122216.24878.1f

24. Kluemper CT, Swafford RE, Hankins MJ, Davis CM, Brzezienski MA and Jemison MD. Flexor tenosynovectomy for recurrent carpal tunnel syndrome: A retrospective case series of 108 hands. Hand 2021;16(1):18-24. https://doi.org/10.1177/1558944719840735

25. Shum C, Parisien M, Strauch RJ and Rosenwasser MP. The role of flexor tenosynovectomy in the operative treatment of carpal tunnel syndrome. J Bone Joint Surg Am. 2002;84(2):221-225. https://doi.org/10.2106/00004623-200202000-00009

26. Gellman H, Gelberman RH, Tan AM and Botte MJ. Carpal tunnel syndrome. An evaluation of the provocative diagnostic tests. J Bone Joint Surg Am. 1986;68(5):735-737.

27. Lew $H L$, Wang $L$ and Robinson LR. Test-retest reliability of combined sensory index: Implications for diagnosing carpal tunnel syndrome. Muscle Nerve. 2000;23(8):1261-1264.

https://doi.org/10.1002/1097-4598(200008)23:8<1261:aidmus $16>3.0 . c 0 ; 2-m$

28. Robinson LR, Micklesen PJ and Wang L. Optimizing the number of tests for carpal tunnel syndrome. Muscle Nerve. 2000;23(12):1880-1882

https://doi.org/10.1002/1097-4598(200012)23:12<1880:aidmus14>3.0.co;2-a

29. American Association of Electrodiagnostic Medicine, American Academy of Neurology and American Academy of Physical Medicine and Rehabilitation. Practice parameter for electrodiagnostic studies in carpal tunnel syndrome: Summary statement. Muscle Nerve. 1993;16:1390-1391.

30. Proubasta IR, Lluch A, Lamas CG, Oller BT and Itarte JP. "Fat Pad" and "Little Finger Pulp" signs are good indicators of proper release of carpal tunnel. Neurosurgery. 2007;61(4):810-814. https://doi.org/10.1227/01.NEU.0000298910.01754.13

31. Levine DW, Simmons BP, Koris MJ, Daltroy LH, Hohl GG, Fossel $\mathrm{AH}$, et al. A self-administered questionnaire for the assessment of severity of symptoms and functional status in carpal tunnel syndrome. J Bone Joint Surg Am. 1993;75(11):1585-1592 https://doi.org/10.2106/00004623-199311000-00002

32. Lin R, Lin E, Engel J and Bubis JJ. Histo-mechanical aspects of carpal tunnel syndrome. Hand. 1983;15(3):305-309.

33. Freshwater MF and Arons MS. The effect of various adjuncts on the surgical treatment of carpal tunnel syndrome secondary to chronic tenosynovitis. Plast Reconstr Surg. 1978;61(1):93-96. https://doi.org/10.1097/00006534-197801000-00016

34. Phalen GS. The carpal-tunnel syndrome. Seventeen years' experience in diagnosis and treatment of six hundred fifty-four hands. J Bone Joint Surg Am. 1966;48(2):211-228.

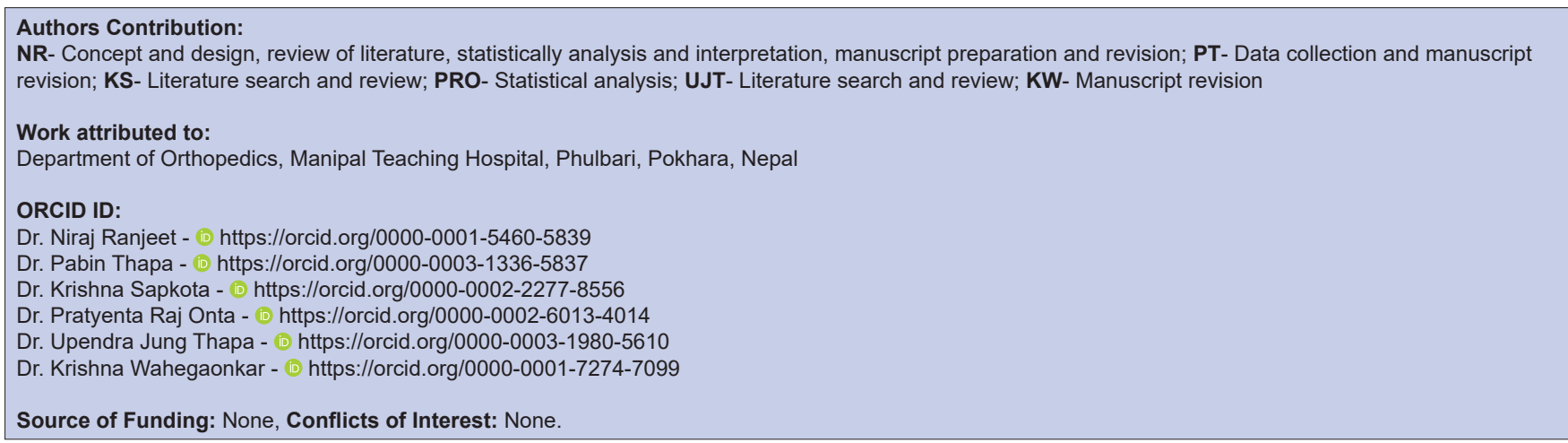

